\title{
Existing Approaches to Smart Parking: An Overview
}

Conference Paper · May 2017

DOI: 10.1007/978-3-319-59513-9_7

CITATIONS

5 authors, including:

Fernando Enriquez

Universidad de Sevilla

43 PUBLICATIONS 297 CITATIONS

SEE PROFILE

Juan Antonio Alvarez-Garcia

Universidad de Sevilla

75 PUBLICATIONS 630 CITATIONS

SEE PROFILE
READS

289

Luis Miguel Soria Morillo

Universidad de Sevilla

32 PUBLICATIONS 382 CITATIONS

SEE PROFILE

Francisco Velasco-Morente

Universidad de Sevilla

129 PUBLICATIONS 794 CITATIONS

SEE PROFILE

Some of the authors of this publication are also working on these related projects:

Project Automatic diatom identification for water quality assessment and monitoring View project

Project Face and facial elements detection View project 


\title{
Existing approaches to smart parking: An overview
}

\author{
Fernando Enríquez ${ }^{1}$, Luis Miguel Soria ${ }^{1}$, Juan Antonio Álvarez-García ${ }^{1}$, Francisco \\ Velasco $^{2}$, and Oscar Déniz ${ }^{3}$ \\ ${ }^{1}$ Computer Languages and Systems Department, University of Seville, 41012 Seville, Spain \\ fenros@us.es,1soria@us.es, jaalvarez@us.es \\ 2 Applied Economics I Department, University of Seville, 41018 Seville, Spain \\ velasco@us.es \\ 3 VISILAB, E.T.S.I.I, University of Castilla-La Mancha, Ciudad Real, Spain \\ oscar.deniz@uclm.es
}

\begin{abstract}
After years of technological advances, parking is still a problem for many people, as a time-consuming task, they have to face on a day-by-day basis, and also for cities, that see how traffic and pollution increases. There have been multiple attempts to find a partial or global technological solution to this problem, ranging from using different types of sensors or cams for automatically detecting free spaces to collaborative apps that let users share related information. In this paper, we give an overview of the methods that have been developed so far, showing their main features, differences, pros, and cons, as well as other factors that may contribute to the success or failure of new proposals that will come in the future.
\end{abstract}

Keywords: Smart city, Parking, Crowdsensing, Computer vision

\section{Introduction}

According to the United Nations [1], 54\% of the population lives in urban zones and this is expected to grow to $66 \%$ in 2050 . This tendency makes urban mobility more difficult and makes finding a parking space one of the most repetitive problems which citizens in big cities have to face up. Furthermore, vehicles cruising for parking are responsible for at least $30 \%$ of traffic jams [2], with the average time for search being more than 20 minutes [3]. Besides the personal problems that this can generate, this is a problem in terms of fuel consumption, $\mathrm{CO} 2$ emissions and in general a waste of resources for the community.

Beyond private spaces, there is no worldwide accepted solution for monitoring vehicles that enter or leave an area, though several efforts have been made to solve the problem in a specific context. Smart parking is the term used for a set of technologies and applications targeting issues related to parking in Smart Cities and this work aims at providing an up-to-date survey of the most interesting and relevant solutions. The rest of the paper is organized as follows. Section 2 analyses the state of the art in Smart Parking solutions. The main conclusions are drawn in Section 3. 


\section{Classification}

Parking solutions are offered to off-street or on-street. Off-street parking may refer to multistory car parks, while on-street refers to parking spaces along public roads and streets. This work considers both types of scenarios. Typically there is a focus on infrastructure, where several sensors are installed to monitor the places, crowdsensing where mobile phones' drivers are the source of the information or a hybrid proposal. Here, vision solutions are analyzed as a specific category due to the increase of cameras and the potential of this type of sensor.

\subsection{Infraestructure}

In order to get the occupancy status of parking places, fixed or mobile sensors are installed in on/off-street parking to detect vehicular events. Mobile sensors are not the most common solution but Parknet [4] in San Francisco is a most prominent study: taxi cars collected the occupancy status of the parking place when they passed beside it, gathering data from GPS receiver and ultrasonic sensors. Although every taxi can detect multiple spots, updating the information of the same spot can take 25 minutes with a fleet of 300 vehicles. Parking Spotter is based on the same idea, leveraging sonar and radar sensors of some Ford vehicles. There are also some LiDAR-based solutions [5] but only focused on surveying parking spaces with one equipped car. These works are hardly scalable since all the vehicles must have the same kind of sensor or else share the information through the same database and the number of mounted sensor-vehicles must be enough to update the information frequently.

Fixed sensors are the more extended and popular option. Although there are a wide variety of sensors [6] (active infrared, ultrasound, acoustic, accelerometer, etc.), magnetometer is by far the most common fixed sensor. The magnetometer is accurate although normally solutions require at least one sensor per place, increasing the cost of the deployment. The sensor itself measures the current magnetic fields and detects the arrival of metal vehicles. Most municipal deployment projects or large shopping centers drill magnetometers on parking places, sharing this information through visual signals or mobile applications. Installation and maintenance processes involve access to the property and road surface so it is only possible for off-street or on-street with government permission.

Table 1 refers to the most prominent smart city parking solutions. SmartSantander and San Francisco can be seen as pilot studies. SmartSantander was conceived as a Smart City Laboratory and San Francisco finished its pilot study at the end of 2013 due to the cost of sensor maintenance. Nice withdrew mobile application Nice Passport in 2016 after some organizational ${ }^{4}$ and security problems ${ }^{5}$. Málaga, London (the only one based on RFID), Moscow or Los Angeles are success cases of Smart Parking.

\footnotetext{
${ }^{4}$ http://www.20minutes.fr/nice/1839579-20160504-nice-trois-ans-apres-installation- stationnement-intelligent-disparait

${ }^{5}$ http://www.lefigaro.fr/politique/le-scan/couacs/2014/06/06/25005-20140606 ARTFIG00112securite-informatique-a-nice-pris-de-court-estrosi-interrompt-une-interview.php
} 
Fixed sensor-based solutions research is focused on reducing the installation time and cost [7] using surface-mounted magnetometers that can be glued to the road and enlarging battery life of wireless sensors.

\begin{tabular}{|l|l|l|l|l|}
\hline Smart City & \#places & Company & URL & Year \\
\hline San Francisco & $6.2 \mathrm{~K}$ & Fybrtech & http://www.fybr-tech.com & 2011 \\
Santander $[8]$ & $0.4 \mathrm{~K}$ & Libelium & http://bit.ly/2mOd38r & 2011 \\
Nice $^{7}$ & $4.5 \mathrm{~K}$ & Urbiotica & http://www.urbiotica.com & 2012 \\
Los Angeles [9] & $6.3 \mathrm{~K}$ & StreetLine & http://lat.ms/1BVDxpD & 2012 \\
London $^{8}$ & $3.4 \mathrm{~K}$ & Smart Parking & http://bit.ly/2mTSTcm & 2012 \\
Moscow $^{9}$ & $50 \mathrm{k}$ & WorldSensing & http://bit.ly/2lCMAbU & 2012 \\
Malaga $^{10}$ & $2.2 \mathrm{~K}$ & Parkhelp & http://bit.ly/2mTPNFc & 2014 \\
\hline
\end{tabular}

Table 1. Infrastructure parking projects

\subsection{Vision}

Due to the increasing interest of the scientific community and users in general by the techniques of artificial intelligence based on images, in recent years there have proliferated solutions to the problem of parking control through systems based on vision. These systems, unlike previous infrastructures, are not yet established, but are mostly used in controlled and experimental environments. Although there are companies that base their systems on these techniques, most of the work is still under development.

In general, the techniques of detection of parking spaces based on vision, suffer from several problems. The first one is the quality of the image. For certain works based on object recognition, the image must be of sufficient quality to be processed. During the day, this may not be an obstacle, but in conditions of insufficient lighting or adverse weather conditions, it becomes a real problem. Another problem related to obtaining the image are occlusions. Depending on the location of the image capture system, the vehicles themselves or surrounding elements (trees, buildings, street furniture, shadows) may obstruct the view of the parking area. This problem can be solved by changing the placement of the capture system, but this may not always be possible, or reduce the monitored surface due to the change in perspective. Finally, an inherent problem with this detection technique is the classification itself. Image-based classification systems have proliferated over the past decade, but they are still far from offering the assurance of systems based on structural elements.

\footnotetext{
${ }^{6}$ http://sfpark.org/resources/parking-sensor-technology-performance-evaluation

${ }^{7} \mathrm{http}$ ///www.urbiotica.com/en/inauguration-of-our-smart-parking-project-in-the-city-of-nice$\mathrm{fr} /$

${ }^{8}$ https://www.westminster.gov.uk/parkright

${ }^{9}$ http://parking.mos.ru/en

${ }^{10} \mathrm{http}: / / \mathrm{www} . e e s c . e u r o p a . e u / ? \mathrm{i}=$ portal.en.events-and-activities-smart-cities-malaga
} 
However, in favor of systems for detecting parking spaces using vision algorithms, there is the coverage, cost, and versatility of the systems. Since a single capture system can cover dozens (or hundreds) of parking spaces, the costs reduction compared to infrastructure-based systems is significant. In addition, the maintenance cost of these systems is negligible, except in those cases where drones or satellites are required to acquire the images. The latter are usually multi-functional, which leads to another strength: versatility. Because the images obtained can be applied not only to the parking control, systems implementing the vision-based solution are often used for other purposes at the same time. Examples of this functionality are surveillance systems, pedestrian control, maintenance tasks, and other scenarios that make the investment made in the implementation of the image capture systems to be quickly amortized.

The parking lots detection solutions based on vision can use different systems to get the images of the zone under control. Among other, the most common methods for the image acquisition are external video cameras, vehicles equipped with vision systems, three-dimensional capture systems, and zenithal or aerial images obtained by satellites or drones.

In the same way, as discussed above, detection systems are supported by a set of algorithms. These algorithms can be grouped by the treatment performed over the images. Most works in the state of the art base their development on some of the following approaches:

- Appearance based approaches. Based on the comparison of the current appearance of a parking place with an original appearance of the vacant state. Many techniques are tailored and fine-tuned to specific contexts and scenarios. However, these techniques can not be easily generalized, and even the adaptation of one solution to a different parking lot is not straightforward.

- Recognition based approaches. Approaches based on object recognition aim to detect and classify the vehicles occupying the parking space using machine learning algorithms. Complex approaches because of the large variety of the target objects.

- Three dimensional image processing.

- Combined techniques applying image processing in order to improve the quality and avoid light variation effects, and machine learning algorithms to classify image content.

Finally, monitoring techniques can be divided into two types depending on the way in which lots are processed:

- Estimating occupancy of an entire parking lot, for example, by counting incoming vehicles.

- Checking for the presence of a vehicle in each cell. Most vision-based approaches require the presence of vehicles in individual parking lots.

Below, a compilation of some relevant papers from the related bibliography are shown. Among them, there are different approaches as a sample of the heterogeneity of the existing processing, recognition, and image acquisition systems.

In [10] is proposed a distributed and efficient system to solve the problem of parking through vision systems. For this purpose convolutional neural networks specifically 
designed for smart cameras are used. Two visual datasets have been used to verify the accuracy of the proposed system: PKLot and CNRPark-EXT. This last dataset was created by the own authors. Thanks to convolutional neural networks applied in the classification process, the proposed solution is robust to images exposed to partial occlusion, shadows, and changes in light conditions. In addition, it has a good capacity of generalization. A reduced version of the AlexNet neural network were used in this paper. The new convolutional neural network is able to recognize only two classes: free or occupied parking space.

Authors in [11] implements a parking detection system based on real-time image processing from video cameras. It divides the system into three sections: image acquisition module, image pre-processing module and image detection module. In the first one, the image is filtered. The detection module is based on the use of a reference image from an empty parking space, without any interference. From this image, converted to grayscale, a comparison system is established with successive images. The algorithm obtains the edges of the reference image and compares them with the last captured. Finally, a function is applied to decide whether the reference image and the compared one have similar characteristics. The accuracy of the system is fair $(81 \%)$.

The solution proposed in [12] uses individual images from a single surveillance camera previously installed in indoor parking lots. This work is also based on reference images from the empty parking lot. The process is slightly different from [11]. The model is generated using Principal Component Analysis, supporting lighting invariance to avoid lighting changes were recognized as potential objects. In addition to the system adaptation to lighting changes, textures are also used to detect objects and isolate them from the background. This makes the system much more robust under extreme lighting changes (large trees, street lamps or intense shadows). In areas with high visibility, the number of occlusions between vehicles is minimized and, hence, systems use to be more accurate. This work has $90 \%$ of accuracy, although it can decrease if occlusions occur in the image.

In [13], the classification is done by a 3-layer Bayesian hierarchical detection Framework (BHDF). To avoid occlusion problems with other cars and surrounding objects, this paper uses the full image for processing, rather than determining the state of the parking spaces one by one. To analyze the whole parking space, the scene is divided into $3 \mathrm{D}$ cubes. Each cube corresponds to a row of car lots. Once the image is obtained and the row of parking spaces to be processed is defined, the BHDF framework is applied. This framework is composed of three pre-trained models, one for the local classification (observation layer), other with the adjacency model (labeling layer) and, the last, with the semantic layer. The local classification model (input model) can be pixel-based or texture-based.

The work presented in [14] deals with the problem of the sunlight, the dark shadows during the day, and the low light intensity at nighttime. To do this, ParkLotD uses a classifier based on the fuzzy c-means clustering algorithm (FCM) and a hyper-parametric fit with particle swarm optimization (PSO). The algorithm has been tested during different hours of the day and under different weather conditions. However, the nighttime use with poor lighting conditions is not advised. At the beginning of the process, it is necessary to define the limits of each parking space, as well as the total number of lots. 
In the next stage, PCA is applied from the feature vectors obtained from each image. Thanks to a user interface, the operator can correct the algorithm operation and show if any error occurs. Furthermore, the operator can retrain the system when needed. The evaluation process was carried out from 2000 images captured by a camera on a roof. Each image covered 27 parking spaces.

In [15], parking states are determined by the combination of an adaptive background subtraction algorithm for moving objects detection (overcoming problems of light changes and shadow effects), with speeded up robust features (SURF) algorithm (robust to scale and rotation changes). Authors propose a solution based on the image appearance and the comparison with a reference image where the lots appear vacant. By using a homogenous transformation to change the point of view, facilitates the extraction of the parking model eliminating perspective distortion. For this process, the user must previously select at least four points that define the parking area. Once the transformation is completed, the parking lots are defined. For the adaptive background subtraction, the Mixture Gaussian Model algorithm is used. This requires a learning process for updating the distributions obtained by the model through a set of images. Once the background is extracted, the characteristics of the images to be classified are obtained. For this process, the algorithms of Speeded Up Robust Features Speeded (invariant to scale changes) and Histogram of Oriented Gradients (HOG) are proposed. The algorithms SVM or KNN can be combined during the classification stage. The authors, through their evaluation with the VI-RAT video database, consider that the best combination of proposed solutions is SURF + SVM, obtaining an average precision of $93 \%$. However, the main problem of this method is the lack of robustness to partial occlusions.

In [16], the aim is not focused on parking areas recognition, but on detecting cars and distances by means of vehicles equipped with a stereo vision system. The authors identify three methods to perform this detection: using radar, using active sensors (laser, radar, lidar) and monocular vision, and finally, using only vision. 2D-based systems may not be enough accurate in identification of the vehicles. To solve this drawback, this system process the 3D image identifying certain potentially characteristics belonging to a vehicle (vertical edges). The algorithm is divided into two main stages: the extraction of three-dimensional characteristics and the detection of the vehicle from them. Extraction of vertical characteristics allows isolating more precisely the obstacles (and vehicles) of the own highway. Two cameras are used for generates 3-D sparse map. As aforementioned, although this kind of algorithm is not specific for parking lots recognition, they can be applied in this context thanks to the capacities to recognize vehicles.

Finally, table 2 shows a schematic representation of some of the most relevant works in parking slots recognition. In table are collected various parameters representative of the different solutions proposed. These parameters include hardware required, algorithm error rate, robustness to lighting changes (low $\bigcirc$, medium $\Theta$, high $\bullet$ ), automation level of the recognition and learning process (manual identification of parking lots for training process $O$, semiautomatic system allowing manual changes in the training process $\theta$, fully-automated system $\bullet$ ), robustness to perspective changes (low $\bigcirc$, medium $\otimes$, high $\bullet$ ), robustness to partial occlusions (low $\bigcirc$, medium $\Theta$, high $\bullet$ ), preferred environment for the system use (indoor, outdoor, both), deployment costs (low $\bigcirc$, medium 
if cameras are already installed $\theta$, complex infrastructure could be required [satellites, drones, ultrasonic sensors] •), computational requirements (low [could be high during the training stage] $\bullet$, medium $\ominus$, high $\bigcirc$ ), scalability (low $\bigcirc$, medium $\ominus$, high $\bullet$ ) and, finally, the way in which the car parks are processed (individually using masks or collectively over the whole area).

\begin{tabular}{|c|c|c|c|c|c|c|c|c|c|c|c|c|}
\hline Author & Year & Error rate & Hardware required & 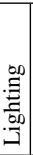 & . & 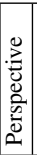 & $\mid \begin{array}{l}0 \\
\vdots \\
. \\
\vdots \\
0 \\
0 \\
0 \\
0\end{array}$ & \begin{tabular}{l}
0 \\
\multirow{0}{0}{} \\
0 \\
0 \\
0 \\
0
\end{tabular} & 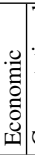 & 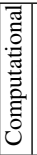 & 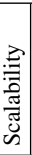 & 节 \\
\hline G. Amato et al. [10] & 2017 & $3 \%$ & Smart-camera & • & $\theta$ & - & - & Both & $\bullet$ & $\theta$ & $\bullet$ & Masks \\
\hline G. Amato et al. [17] & 2016 & $9 \%$ & Smart-camera + RPi & - & Q & - & $\bullet$ & Both & - & Q & - & Masks \\
\hline I. Masmoudi et al. [15] & 2016 & $7 \%$ & Videocamera & $\theta$ & Q & $\theta$ & 0 & Outdoor & $\theta$ & Q & $\theta$ & Masks \\
\hline J. Suhr et al. [18] & 2014 & $1.90 \%$ & AVM and ultrasound & - & - & - & $\bullet$ & Both & 0 & $\bullet$ & 0 & Vehicle \\
\hline J. Jermsurawong et al. [19] & 2012 & $1 \%$ & Videocamera & • & Q & 0 & $\bullet$ & Outdoor & Q & Q & - & Full area \\
\hline H. Ichihashi et al. [14] & 2009 & $7 \%$ & Videocamera + server & $\theta$ & Q & 0 & 0 & Both & $\theta$ & Q & 0 & Masks \\
\hline Z. Bin et al. [11] & 2009 & $19 \%$ & Video camera + CPU & 0 & 0 & 0 & 0 & Both & $\theta$ & - & $\theta$ & Masks \\
\hline C. Huang et al. [13] & 2008 & $6 \%$ & IP camera + CPU & $\theta$ & $\theta$ & 0 & $\bullet$ & Indoor & $\theta$ & Q & $\theta$ & Rows \\
\hline G. Toulminet et al. [16] & 2006 & N/D & Stereo-vision system $+\mathrm{CPU}$ & - & - & - & 0 & Outdoor & $\theta$ & Q & 0 & Vehicle \\
\hline Q. Zhang et al. [20] & 2006 & $20 \%$ & Satellite images & - & - & - & $\bullet$ & Outdoor & 0 & - & 0 & Full area \\
\hline S. Funck et al. [12] & 2004 & $10 \%$ & CCTV-cameras & Q & Q & 0 & 0 & Indoor & $\theta$ & - & Q & Masks \\
\hline K. Yamada et al. [21] & 2001 & $0.60 \%$ & Videocamera & $\theta$ & $\theta$ & - & 0 & Outdoor & $\theta$ & - & $\theta$ & Masks \\
\hline X. Wang et al. [22] & 1998 & $25 \%$ & Aerial images & - & Q & - & - & Outdoor & 0 & - & 0 & Full area \\
\hline
\end{tabular}

Table 2. Vision-based parking lot detection algorithms

\subsection{Social crowdsensing}

Using software applications to help drivers park their vehicles is not new, even with solutions that include the location, reservation, access and payment tasks all together as in [23]. Nevertheless, the growing popularity of smartphones in the last years, full of sensors and capable of registering geo-positional information in short periods of time, has created new ways of searching for a parking spot favoring the rise of multiple mobile and web applications. Table 3 shows a comparison between some of the apps for on-street or off-streets parking we can find on the Internet. The selection criteria used to filter the huge catalog that exists nowadays is based on the popularity, but also on the special features offered by some of them. Most of the apps are centered uniquely on off-street parking, maybe collecting data from installed sensors or cams. Parkopedia (available worldwide) or wesmartPark (working in Madrid and Barcelona), are different examples of these off-street parking solutions. The latter offers the installation of sensor technology and a management module for owners to easily monitor their business while they obtain data for their app users. While the basic operations in these apps remain the same, we find interesting proposals in the literature that could change the scene someday, i.e. bringing automatic price negotiation capabilities [24]. There are also apps that give information of on-street parking, recommending the best zones to search for unoccupied parking spots, or giving the opportunity to announce when a user is about to move a vehicle, leaving a free space, and in some cases, allowing reservations for this 
new free spots. Each of these functionalities can use data obtained automatically by cell phones (crowdsensing) or introduced manually by the app users (crowdsourcing). This approach, automatic or manual, is particularly useful for on-street parking, where the scalability of other approaches is more difficult due to the size of the areas to be covered. While off-street parking information can primarily help users reduce the price to pay or select the closest space to their destination, on-street parking information usually has as its main goal the mitigation of the "multiple-car-chasing-single-space" phenomenon [25] derived from the common "blind search" strategy.

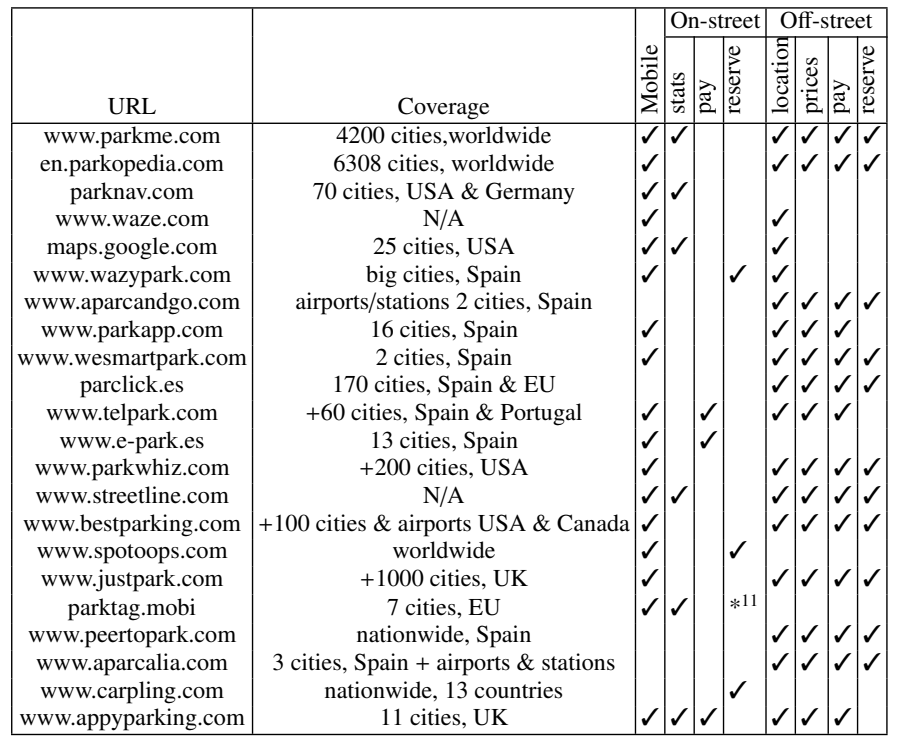

Table 3. Smart parking apps

When the information is retrieved in an automatic way, it is usually visualized as a statistical estimation, showing the street maps with different colors indicating whether it is easy, medium or difficult to find parking spots. This coarse grain classification tags can help drivers heading towards zones where it is more likely to be successful but it obviously does not guarantee an available parking space. This analysis is based on the processing of data (i.e. cell phone signals) in a massive way, generating mathematical models that represent global data, just like it is done to show the traffic density during different hours of the day. ParkMe (owned by INRIX), Parknav, Streetline and Google Maps, are examples of parking solutions that employ machine learning algorithms to process automatically retrieved parking data. Streetline is heavily focused on data analytics and offers different solutions, being "Parker" the one that shows real time parking

${ }^{11}$ Planned 
availability to the user. In the case of Google Maps, it is a new functionality ${ }^{12}$ only available in some US cities.

Related with this big data perspective, we can find research on related tasks that can individually detect parking activity. In order to detect when a car has been parked or when its location is going to become a free spot in a near future, algorithms can focus on activity transition detection, from driving to walking and vice versa, or on the prediction of user destinations, to find out for example if a user is walking towards the vehicle. In [26] we find an example of automatic detection of parking availability through what the authors call "pocketsourcing". Monitoring the activities using phone sensors result in a $94 \%$ of correctly predicted parking availability according to their experiments. ParkTAG uses this kind of technology with a so-called "Auto-detection algorithm" and is actually working in seven European cities. The main obstacle is to convince users to keep the app running on background on their devices. At the beginning, there is a lack of users executing the algorithm, which results in incomplete information and low accuracy of the outcome. This is referred to as the cold start problem that affects all the crowdsource initiatives, forcing us to achieve a critical mass of users for the project to be viable. Other research papers, like [27], focus on the optimization of the overall parking process, considering a cost function that depends on the proximity to destination and the parking price.

Another approach is to focus on the information systems that access the in-car sensor-network present in modern vehicles. Some of the mentioned methods would also apply in this scenario, although with some limitations, as we have access to plenty of data from the car, but not from the user. Therefore, it makes easier to analyze the movement profile of the car but does not include the user movements, leaving out valuable information for example to predict parking status changes based on user habits or real time monitoring. From a general point of view, the main problem of this approach is the lack of a unified database to share the information coming from vehicles of different brands, which makes it necessary to work with external data from other sources. An example is the BMW on-street parking information system, incorporated in cars of this brand manufactured in Germany or the United States since last year, which uses the INRIX parking information database. BMW is also present in other solutions like JustPark after a remarkable investment. It is also possible to extend the technology already provided by the car manufacturer installing extra equipment, as the appyparking team is suggesting for example. Installing a M2M dongle in any car will enable the "one click parking" they have developed, allowing semi-automatic payment just confirming the location on our phone.

Finally, among the apps currently available in Android and iOS marketplaces, we find collaborative apps that allow "manual" sharing of parking information. People announce when they are about to leave, so that other users can drive towards their location and occupy that space. Some of these apps also offer a reservation feature, which forces the outgoing car to publish the information with enough time for its counterpart to arrive, or to wait until the operation is done. The mentioned Parknav app has the 'I'm leaving" feature, which is used in their real time parking information system, and ParkTAG claims they will incorporate this feature soon. Others are directly focused on this

\footnotetext{
${ }^{12}$ https://blog.google/products/maps/know-you-go-parking-difficulty-google-maps/
} 
feature, like spotoops, which is a clear example of the major drawback of this approach, the cold start problem once again. The app is available worldwide, but it does not have enough users, what makes it useless in most cases having no spots offered in the zone we are interested in. Even when the app has a large number of users, it is necessary to implement ways to stimulate the participation of those users and also to encourage them to contribute high quality data [28]. Another app that is trying to overcome this obstacle is Wazypark, first narrowing the scope (it is only available in Spain) and also offering other features to attract more users (like a comparator of gas stations), which seems to be working as the number of users keep growing. Carpling is another collaborative application where parking spots is just one of the assets (car, taxi, train,...) people can share, becoming a collaborative social network. The public condition of on-street parking makes some people disagree with the offer-demand concept of these apps, especially when the user that offers a spot sets a price to pay for it. In 2014, people started publishing negative opinions about this type of technological help that works against those who stay out of these new-born communities, the so-called "freeriders". In fact, the term "jerktech" was created in San Francisco to talk about those startups that make profit by exploiting small businesses and public infrastructure favoring the rich that can pay for their services (MonkeyParking was one of the main victims of this opinion wave). Keeping the monetary aspect aside, there have been some studies showing that parking apps can benefit not only their users, but the entire community, including "freeriders" [29].

\section{Discussion}

In this paper, the main approaches to the problem of car park recognition have been presented in a general way. Three types of systems have been differentiated: those based on infrastructure, those based on vision and those based on crowdsensing. Each one of them presents advantages and disadvantages that must be faced during the next years. In the case of systems based on infrastructure, the main limitation is the cost of implementing such solutions. In addition, the cost of maintenance is also one of the main reasons why many infrastructure-based systems have failed to succeed.

On the other hand, the vision-based systems have proliferated in the last decade, reason why the techniques of processing of images still do not obtain an excellent result under certain conditions. The main problems faced by these techniques are lighting changes, partial occlusions and generalization capacity. However, the cost of deployment and maintenance is much lower than in infrastructure-based solutions.

Finally, due to the expansion of social systems, crowdsensing techniques are a clear alternative for the future to solve the parking problem. However, because of their lack of maturity and the problems inherent in social systems, their implementation still does not end up being a reality in the vast majority of cases. The problems of cold start or community involvement require solutions to turn crowdsensing-based systems into an effective alternative to infrastructure or vision based systems. With the support of smartphones software giants, this could become a reality. 


\section{Acknowledgements}

This research is partially supported by the Spanish Economy Ministry and FEDER R\&D through the "HERMES-Smart Citizen" project (TIN2013-46801-C4-1-R).

\section{References}

1. John Bongaarts. United nations department of economic and social affairs, population division world mortality report 2005. Population and Development Review, 32(3):594-596, 2006.

2. Donald C Shoup. Cruising for parking. Transport Policy, 13(6):479-486, 2006.

3. S Gallivan. Ibm global parking survey: Drivers share worldwide parking woes technical report. Technical report, Technical report, IBM, 2011.

4. Suhas Mathur, Tong Jin, Nikhil Kasturirangan, Janani Chandrasekaran, Wenzhi Xue, Marco Gruteser, and Wade Trappe. Parknet: drive-by sensing of road-side parking statistics. In Proceedings of the 8th international conference on Mobile systems, applications, and services, pages 123-136. ACM, 2010.

5. Fabian Bock, Daniel Eggert, and Monika Sester. On-street parking statistics using lidar mobile mapping. In Intelligent Transportation Systems (ITSC), 2015 IEEE 18th International Conference on, pages 2812-2818. IEEE, 2015.

6. Trista Shuenying Lin. Smart parking: Network, infrastructure and urban service. $\mathrm{PhD}$ thesis, Lyon, INSA, 2015.

7. Simon Evenepoel, Jan Van Ooteghem, Sofie Verbrugge, Didier Colle, and Mario Pickavet. On-street smart parking networks at a fraction of their cost: performance analysis of a sampling approach. Transactions on Emerging Telecommunications Technologies, 25(1):136149, 2014.

8. Luis Sanchez, Luis Muñoz, Jose Antonio Galache, Pablo Sotres, Juan R Santana, Veronica Gutierrez, Rajiv Ramdhany, Alex Gluhak, Srdjan Krco, Evangelos Theodoridis, et al. Smartsantander: Iot experimentation over a smart city testbed. Computer Networks, 61:217-238, 2014.

9. Peer Ghent. Optimizing performance objectives for projects of congestion pricing for parking. Transportation Research Record: Journal of the Transportation Research Board, (2530):101-105, 2015.

10. Giuseppe Amato, Fabio Carrara, Fabrizio Falchi, Claudio Gennaro, Carlo Meghini, and Claudio Vairo. Deep learning for decentralized parking lot occupancy detection. Expert Systems with Applications, 72:327-334, 2017.

11. Zhang Bin, Jiang Dalin, Wang Fang, and Wan Tingting. A design of parking space detector based on video image. ICEMI 2009 - Proceedings of 9th International Conference on Electronic Measurement and Instruments, pages 2253-2256, 2009.

12. Stefan Funck, Nikolaus Mohler, and Wolfgang Oertel. Determining car-park occupancy from single images. In Intelligent Vehicles Symposium, 2004 IEEE, pages 325-328. IEEE, 2004.

13. Ching-chun Huang, Sheng-jyh Wang, Yao-jen Chang, and Tsuhan Chen. A bayesian hierarchical detection framework for parking space detection. IEEE International Conference on Acoustics, Speech and Signal Processing, 2008. ICASSP 2008, 1:2097-2100, 2008.

14. Hidetomo Ichihashi, Akira Notsu, Katsuhiro Honda, Tatsuya Katada, and Makoto Fujiyoshi. Vacant parking space detector for outdoor parking lot by using surveillance camera and fcm classifier. In Fuzzy Systems, 2009. FUZZ-IEEE 2009. IEEE International Conference on, pages 127-134. IEEE, 2009. 
15. Imen Masmoudi, Ali Wali, Anis Jamoussi, and Adel M Alimi. Vision based system for vacant parking lot detection : Vpld. IEEE International Conference on Computer Vision Theory and Applications (VISAPP), Vol. 2., 2014., (January 2014):1-8, 2016.

16. Gwenaëlle Toulminet, Massimo Bertozzi, Stéphane Mousset, Abdelaziz Bensrhair, and Alberto Broggi. Vehicle detection by means of stereo vision-based obstacles features extraction and monocular pattern analysis. IEEE Transactions on Image Processing, 15(8):2364-2375, 2006.

17. Giuseppe Amato, Fabio Carrara, Fabrizio Falchi, Claudio Gennaro, Claudio Vairo, and G Moruzzi. Car parking occupancy detection using smart camera networks and deep learning. IEEE Symposium on. IEEE Computers and Communication (ISCC), 2016., (D1), 2016.

18. Jae Kyu Suhr and Ho Gi Jung. Sensor fusion-based vacant parking slot detection and tracking. IEEE Transactions on Intelligent Transportation Systems, 15(1):21-36, 2014.

19. Jermsak Jermsurawong, Mian Umair Ahsan, Abdulhamid Haidar, Haiwei Dong, and Nikolaos Mavridis. Car parking vacancy detection and its application in 24-hour statistical analysis. In Frontiers of Information Technology (FIT), 2012 10th International Conference on, pages 84-90. IEEE, 2012.

20. Qiaoping Zhang and Isabelle Couloigner. Benefit of the angular texture signature for the separation of parking lots and roads on high resolution multi-spectral imagery. Pattern Recognition Letters, 27(9):937-946, 2006.

21. Keiichi Yamada and Morimichi Mizuno. A vehicle parking detection method using image segmentation. Electronics and Communications in Japan (Part III: Fundamental Electronic Science), 84(10):25-34, 2001.

22. Xiaoguang Wang and Allen R Hanson. Parking Lot Analysis and Visualization from Aerial Images. In Fourth IEEE Workshop on Applications of Computer Vision, 1998.

23. Thomas B Hodel and Suo Cong. Parking space optimization services, a uniformed web application architecture. In ITS World Congress Proceedings, pages 16-20, 2003.

24. Shuo-Yan Chou, Shih-Wei Lin, and Chien-Chang Li. Dynamic parking negotiation and guidance using an agent-based platform. Expert Systems with Applications, 35(3):805-817, 2008.

25. Pu Wang, Timothy Hunter, Alexandre M Bayen, Katja Schechtner, and Marta C González. Understanding road usage patterns in urban areas. arXiv preprint arXiv:1212.5327, 2012.

26. Anandatirtha Nandugudi, Taeyeon Ki, Carl Nuessle, and Geoffrey Challen. Pocketparker: Pocketsourcing parking lot availability. In Proceedings of the 2014 ACM International Joint Conference on Pervasive and Ubiquitous Computing, pages 963-973. ACM, 2014.

27. Yanfeng Geng and Christos G Cassandras. New "smart parking" system based on resource allocation and reservations. IEEE Transactions on Intelligent Transportation Systems, 14(3):1129-1139, 2013.

28. Baik Hoh, Tingxin Yan, Deepak Ganesan, Kenneth Tracton, Toch Iwuchukwu, and JuongSik Lee. Trucentive: A game-theoretic incentive platform for trustworthy mobile crowdsourcing parking services. In Intelligent Transportation Systems (ITSC), 2012 15th International IEEE Conference on, pages 160-166. IEEE, 2012.

29. Xiao Chen, Elizeu Santos-Neto, and Matei Ripeanu. Crowdsourcing for on-street smart parking. In Proceedings of the second ACM international symposium on Design and analysis of intelligent vehicular networks and applications, pages 1-8. ACM, 2012. 